\title{
Tacit Knowledge in the Workplace
}

\author{
Robert J. Sternberg \\ Yale University
}

\author{
George B. Forsythe \\ United States Military Academy \\ Joseph A. Horvath \\ IBM Consulting Group \\ Scott Snook \\ United States Military Academy \\ Richard K. Wagner \\ Florida State University
}

\author{
Jennifer Hedlund \\ Yale University \\ Trueman Tremble \\ U.S. Army Research Institute \\ Wendy M. Williams \\ Cornell University \\ Elena L. Grigorenko \\ Yale University
}

\section{Ft. Leavenworth Research Unit Stanley M. Halpin, Chief}
U.S. Army Research Institute for the Behavioral and Social Sciences 5001 Eisenhower Avenue, Alexandria, Virginia 22333-5600

March 1999

Army Project Number

20262785A790
Personnel Systems and Performance Technology

Approved for public release; distribution is unlimited. 